INPLASY

PROTOCOL

To cite: Guo et al. Massage

Therapy for Athletic Injuries:

Systematic Review and Meta-

Analysis. Inplasy protocol

202160043. doi:

10.37766/inplasy2021.6.0043

Received: 14 June 2021

Published: 14 June 2021

Corresponding author:

Min Fang

fangmin19650510@163.com

Author Affiliation:

School of Acupuncturemoxibustion and Tuina, Shanghai University of

Traditional Chinese Medicine, Shanghai 201203, China.

Support: University.

Review Stage at time of this submission: Piloting of the study selection process.

\section{Massage Therapy for Athletic Injuries: Systematic Review and Meta-Analysis}

Guo, G1; Xie, S2; Xu, J3; Zhou, X4; Huang, F5; Wu, B6; Wu, G7; Zhang, Y8; Yao, F9; Shan, C10; Fang, $\mathrm{M}^{11}$.

Review question / Objective: The aim of this systematic review and meta-analysis of randomized controlled trials is to evaluate the efficacy and safety of massage therapy for athletic injuries.

Condition being studied: Athletic injuries. Massage Therapy. Information sources: We will search in PubMed, the Cochrane Library, EMBASE and four Chinese databases (CNKI, Wan Fang, CBMdisc, and VIP) for articles published through June 2021. The search string will be built as follows: (massage OR massage therapy) AND (athletic injuries OR sports injuries). The electronic database search will be supplemented by a manual search of the reference lists of included articles.

INPLASY registration number: This protocol was registered with the International Platform of Registered Systematic Review and Meta-Analysis Protocols (INPLASY) on 14 June 2021 and was last updated on 14 June 2021 (registration number INPLASY202160043).

Conflicts of interest:

None declared.

\section{INTRODUCTION}

Review question / Objective: The aim of this systematic review and meta-analysis of randomized controlled trials is to evaluate the efficacy and safety of massage therapy for athletic injuries.
Condition being studied: Athletic injuries. Massage Therapy.

\section{METHODS}

Participant or population: Inclusion criteria: Adults with athletic injuries (as diagnosed by a clinician, or using any recognized diagnostic criteria) will be included. 
Exclusion criteria: Severe primary disease such as cardiovascular, lung, kidney, and hematopoietic disease, pregnant woman, patients with mental illness.

Intervention: Massage was the main intervention (e.g. Chinese massage, and Thai massage).

\section{Comparator: Not applicable.}

Study designs to be included: We will include randomised trials to assess the beneficial effects of the treatments, and will supplement these with observational studies for the assessment of harms.

Eligibility criteria: Therapeutical massage is defined as the manipulation of the soft tissue of whole body areas to bring about generalised improvements in health, such as relaxation or improved sleep, or specific physical benefits, such as relief of muscular aches and pains. We included RCTs, trials were eligible if the intervention included at least 1 massage, more than 20 subjects in each group, and original data. Studies that accord with the lastest diagnostic criteria were eligible.

Information sources: We will search in PubMed, the Cochrane Library, EMBASE and four Chinese databases (CNKI, Wan Fang, CBMdisc, and VIP) for articles published through June 2021. The search string will be built as follows: (massage OR massage therapy) AND (athletic injuries OR sports injuries). The electronic database search will be supplemented by a manual search of the reference lists of included articles.

Main outcome(s): Change in pain score from baseline to the last available followup, measured using the visual analogue scale.

Quality assessment / Risk of bias analysis: The risk of bias for each of the following domains was assessed for each study: (1) random sequence generation, (2) allocation concealment, (3) blinding of the participants and personnel, (4) blinding of the outcome assessments, (5) incomplete outcome data, (6) selective reporting, and (7) other bias. Each study included was rated as having a high, low, or unclear risk of bias. Two authors (CFH and XSJ) evaluated all the data extracted and quality ratings for consistency and resolved disagreements. Disagreements were resolved by discussion with a third author (WBY).

Strategy of data synthesis: We qualitatively synthesized all the included studies The included studies on pain were synthesized based on the VAS pain score and the WOMAC pain score separately. The VAS score ranged from 0 points (no pain) to 10 points (worst possible pain). The WOMAC pain score ranged from 0 points to 20 points, with a lower score representing a better outcome. All analyses were conducted using RevMan V5.4. For the meta-analysis of the VAS pain score, WOMAC pain score, etc, we combined studies using the mean difference (MD); a positive MD indicated that the effect of massage therapy was favourable compared with the control therapy. For the total effectiveness rate, we combined studies using the risk ratio (RR) in the meta-analysis, and an RR of the total effectiveness rate greater than 1 indicated that massage was more effective than was the control therapy. We evaluated heterogeneity using the 12 statistic. P values $<0.05$ were considered to indicate statistical significance in all the results.

Subgroup analysis: Patients with sports injuries in China and other regions will be included in the covariate survey. We will compare the effective rate of Chinese massage with Other massage techniques by observing visual analogue scale pain score.

Sensitivity analysis: We will choose different statistical models and subgroup analysis, or exclude low-quality literature to explore possible sources of bias.

Country(ies) involved: China.

Keywords: Massage; Athletic Injuries; Muscle; Jionts. 
Contributions of each author:

Author 1 - Guangxin Guo.

Author 2 - Shengji Xie.

Author 3 - Jianghan Xu.

Author 4 - Xu Zhou.

Author 5 - Feihong Huang.

Author 6 - Boyi Wu.

Author 7 - Guanghui Wu.

Author 8 - Yan Zhang.

Author 9 - Fei Yao.

Author 10 - Chunlei Shan.

Author 11 - Min Fang. 